\title{
Adaptive Robust State Observers of Uncertain Dynamical Systems with Time-Varying Delays
}

\author{
Hansheng $\mathrm{Wu}$ \\ Department of Information Science, Prefectural University of Hiroshima \\ Hiroshima City, Hiroshima 734-8558, Japan \\ hansheng@pu-hiroshima.ac.jp
}

\begin{abstract}
The problem of adaptive robust state observer design is considered for a class of uncertain dynamical systems with Time-varying delays. A new method is presented whereby a class of memoryless adaptive robust state observers with simpler structure is proposed. It is also shown that by employing the proposed adaptive robust state observer, the observation error between the observer state estimate and the true state can be guaranteed to be uniformly exponentially convergent towards a ball which can be as small as desired. Finally, a numerical example is given to demonstrate the validity of the results.
\end{abstract}

Keywords-Adaptive control; robust control; time-delay systems; state observer; convergence

\section{Introduction}

It is well known that the problem of robust state observer design for dynamical systems with significant uncertainties has received considerable attention of many researchers, and some approaches to designing a robust state observer has been developed in the past decades (see, e.g. [1]-[7] for time--delay systems). Here, it is worth pointing out that the time delays are often assumed to be known, and such delays are employed to construct some types of state observers, in most of works which are concerned with state observer design problem of time-delay dynamical systems. Moreover, in the control literature on time-delay dynamical systems, the terms including delayed state variables are generally assumed either to be linear or to be linear norm-bounded in the states.

On the other hand, for dynamical systems with significant uncertainties, the upper bounds of the vector norms on the uncertainties are generally supposed to be known, and such bounds are employed to construct some types of robust state observers (see, e.g. [1,2,3] or [6] for time-delay systems). However, in a number of practical control problems, such bounds may be unknown, or be partially known. Therefore, for such a class of uncertain dynamical systems, an adaptive scheme should be introduced to update these unknown bounds to construct some types of robust state observers. In general, such an observer is called adaptive robust state observer.

In the past decades, some efforts have been made to consider the problem of adaptive robust state observer for uncertain dynamical systems with the unknown bounds of uncertainties or perturbations (see, e.g. [8,9] and the references therein). In particular, in some recent papers, the problem of adaptive robust state observer is also considered for uncertain time-delay systems. In [10], for example, the problem of adaptive robust state observer design is considered for a class of uncertain systems with delayed state perturbations.
In this paper, similar to [10], we also consider the problem of adaptive robust state observer design for a class of uncertain nonlinear time-delay systems. However, being different from [10], we assume that the time-varying delays are any nonnegative continuous and bounded functions which do not require that their derivatives have to be less than one. We present a new method whereby a class of continuous memoryless adaptive robust state observers with a rather simpler structure is proposed. We also show that the proposed adaptive robust state observers can guarantee that the observation error between the observer state estimate and the true state converges uniformly exponentially towards a ball which can be as small as desired. In addition, because the adaptive robust state observers proposed in the paper are completely independent of time delays, the results obtained here may be applicable to a class of dynamical systems with uncertain time delays.

The paper consists of the following parts. In Section 2, the state observation problem to be tackled is stated and some standard assumptions are introduced. In Section 3, a class of memoryless adaptive robust state observers is proposed and the corresponding convergence analysis is made. In Section 4, a numerical example is given to illustrate the validity of the results. Finally, the paper concludes in Section 5 with a brief discussion of the results obtained in the paper.

\section{Problem Formulation}

We consider a class of uncertain dynamical systems with time-varying delay described by the following differentialdifference equations:

$$
\frac{d x(t)}{d t}=A x(t)+B u(t)+\Delta f(x(t-h(t))
$$




$$
y(t)=C x(t)
$$

where $t \in R$ is the time, $x(t) \in R^{n}$ is the current value of the state, $u(t) \in R^{m}$ is the control vector, $y(t) \in R^{p}$ is the output vector, $A, B, C$ are constant matrices of appropriate dimensions, and the nonlinear function $\Delta f(\cdot)$ represents the delayed state perturbations and is assumed to be continuous in all their arguments. Moreover, the constant matrix $C$ is assumed to be of full rank. In addition, the time delay $h(t)$ is assumed to be any continuous bounded function satisfying

$$
0 \leq \bar{\tau} \leq h(t) \leq \bar{h}
$$

where $\bar{\tau}$ and $\bar{h}$ are any nonnegative constants, and $\bar{\tau}$ is not required in general to be zero. It is worth noting that the function $\mathrm{h}(\mathrm{t})$ is not required to be known for the system designer.

The initial condition for system (1) is given by

$$
x(t)=\chi(t), \quad t \in\left[t_{0}-\bar{h}, \quad t_{0}\right]
$$

where $\chi(t)$ is a given continuous function.

Now, the question is how to design a continuous state observer with the output $y(t)$ and input $u(t)$ such that the state estimate $\hat{x}(t)$ can converge to the original state $\mathrm{x}(\mathrm{t})$, as far as possible, in the presence of the delayed state perturbations.

Before proposing our state observers, we introduce for system (1) the following assumptions.

Assumption 2.1. The pair ( $A, C)$ given in system (1) is completely observable. That is, there exists a matrix $\mathrm{K} \in \mathrm{R}^{\wedge}\{\mathrm{n} \times \mathrm{p}\}$ such that the matrix $A_{0}:=A-K C$ is Hurwitz.

Assumption 2.2. There exists a vector nonlinear function $\xi(\cdot): R^{n} \rightarrow R^{p}$ such that the following matching condition can be satisfied

$$
\Delta f(x(t-h(t)))=P^{-1} C^{T} \xi(x(t-h(t)))
$$

where the positive definite matrix $\mathrm{P}$ is the unique solution to the Lyapunov equation of the form

$$
A_{0}^{T} P+P A_{0}=-Q
$$

for any given positive definite matrix $Q \in R^{n \times n}$.
Assumption 2.3. The uncertain function $\xi(\cdot)$ is bounded in Euclidean norm. Moreover, there exists an unknown constant $\theta^{*} \in R^{+}$such that for any $t \geq 0$, we have

$$
\|\xi(x(t-h(t)))\| \leq \theta^{*}\|x(t-h(t))\|
$$

Without loss of generality, we introduce the following definition:

$$
\psi^{*}:=\varepsilon \eta^{-1}\left(\theta^{*}\right)^{2}
$$

where $\eta$ is any given positive constant, and $\varepsilon$ is any positive constant which is not required to be known for the system designer. It is obvious from Assumption 2.3 that $\psi^{*}$ is still an unknown positive constant.

Remark 2.1. Assumption 2.2 represents that the uncertain time-delay dynamical systems, described by (1), have a special structure which is generally called a matching condition about the nonlinearity and uncertainty, and is a rather standard assumption for the problem of robust state observers. In fact, in number of practical control systems, particularly mechanical systems, environmental systems, ecological systems, industrial electronic systems, and so on, such a condition is often satisfied (see, e.g. [1,2], [8]-[10], and the references therein). In addition, Assumption 2.3 defines the uncertainty bands (in general state or output dependent).

\section{Main Results}

In this section, we propose a class of continuous adaptive robust state observers with a rather simple structure, which is independent of the time delays and bounds of nonlinear perturbations, such that the state estimate $\hat{x}(t)$ can converge as far as possible to the original state $x(t)$. For this, the observation error between the observer state estimate and the true state is defined as

$$
e(t)=x(t)-\hat{x}(t)
$$

Now, for the uncertain time-delay dynamical systems described by (1) we propose the following adaptive robust state observer without time delays:

$$
\begin{aligned}
\frac{d \hat{x}(t)}{d t}=A \hat{x}(t) & +B u(t)+K(y(t)-\hat{y}(t)) \\
+ & P^{-1} C^{T} E(\hat{x}(t), y(t), \hat{\psi}(t), t)
\end{aligned}
$$

where $\hat{x}(t) \in R^{n}$ is the state estimate vector, $\hat{y}(t) \in R^{p}$ is the output vector of the observer, $K \in R^{n \times p}$ 
is constant matrix, called the gain matrix, and $E(\hat{x}(t), y(t), \hat{\psi}(t), t)$ is an auxiliary vector function which will be given by

$$
E(\hat{x}(t), y(t), \hat{\psi}(t), t)=\frac{1}{2} \eta \hat{\psi}(t) C e(t)
$$

In particular, the time function $\hat{\psi}(t) \in R^{+}$in (8) is the estimate of the unknown parameter $\psi^{*} \in R^{+}$which is updated by the following adaptation law:

$$
\frac{d \hat{\psi}(t)}{d t}=-\gamma \sigma \hat{\psi}(t)+\gamma \eta\|C e(t)\|^{2}
$$

where $\sigma$ and $\gamma$ are any given positive constants.

Thus, it is obvious from (1) and (8) that we can easily obtain the observation error time-delay dynamical systems of the form:

$$
\begin{aligned}
\frac{d e(t)}{d t}=A_{0} e(t) & +\Delta f(x(t-h(t))) \\
& -P^{-1} C^{T} E(\hat{x}(t), y(t), \hat{\psi}(t), t)
\end{aligned}
$$

On the other hand, letting $\tilde{\psi}(t)=\hat{\psi}(t)-\psi^{*}$, we can rewrite (9) as the following adaptation error systems:

$$
\frac{d \tilde{\psi}(t)}{d t}=-\gamma \sigma \tilde{\psi}(t)+\gamma \eta\|C e(t)\|^{2}-\gamma \sigma \psi^{*}
$$

In the following, by the pair $(e(t), \tilde{\psi}(t))$ we denote the solutions to the time-delay error systems described by (10) and (11). Thus, we have the following theorem which shows that the state estimate $\hat{x}(t)$ of adaptive robust observer (8) with the estimate $\hat{\psi}(t)$ given in updating law (9) can converge as far as possible to the original state $x(t)$.

Theorem 3.1. Consider the time-delay error systems described by (10) and (11) satisfying Assumptions 2.1 to 2.3. Then, the solutions $(e, \widetilde{\psi})\left(t ; t_{0}, e\left(t_{0}\right), \widetilde{\psi}\left(t_{0}\right)\right)$ to (10) and (11) are uniformly bounded and the error $e(t)$ converges uniformly exponentially towards a ball which can be as small as desired, in the presence of the nonlinear timevarying delayed state perturbations.

Proof: For the adaptive time-delay error systems described by (10) and (11), we first define a quasi-Lyapunov functional as follows.

$$
V(e, \widetilde{\psi})=e^{T}(t) P e(t)+\frac{1}{2} \gamma^{-1} \widetilde{\psi}^{2}(t)
$$

where $P$ is the solution of Lyapunov equation (5), and $\gamma$ is any positive constant.

Let $(e(t), \widetilde{\psi}(t))$ be the solution of the time-delay error systems described by (10) and (11) for any $t \geq t_{0}$. Then, by taking the derivative of $V(\cdot)$ along the trajectories of (10) and (11), from Assumption 2.2 and Assumption 2.3 it can be obtained that for any $t \geq t_{0}$,

$$
\begin{aligned}
\frac{d V(e, \tilde{\psi})}{d t} \leq & -e^{T}(t) Q e(t)+2 \theta^{*}\|c e(t)\|\|x(t-h(t))\| \\
& -2 e^{T}(t) C^{T} E(\hat{x}(t), y(t), \hat{\psi}(t), t) \\
& +\gamma^{-1} \tilde{\psi}(t) \frac{d \tilde{\psi}(t)}{d t}
\end{aligned}
$$

After some trivial manipulations, from (13) we can obtain that for any $t \geq t_{0}$,

$$
\|e(t)\|^{2} \leq \frac{\lambda_{\min }^{-1}(P)}{1-\kappa^{*}} V\left(t_{0}\right) e^{\bar{\theta}\left(t-t_{0}\right)}+\frac{\lambda_{\min }^{-1}(P) \bar{\varepsilon} \alpha^{-1}}{1-\kappa^{*}}
$$

where $\bar{\theta}, \bar{\varepsilon}, \alpha$, and $\kappa^{*}$ are any positive constants.

Then, from (14) we know that $\|e(t)\|$ is uniformly bounded, and converges uniformly exponentially to a ball $\mathrm{B}(\delta)$ where

$$
\mathrm{B}(\delta):=\left\{e \mid\|e(t)\| \leq \delta=\sqrt{\lambda_{\min }^{-1}(P) \bar{\varepsilon} \alpha^{-1} /\left(1-\kappa^{*}\right)}\right\}
$$

On the other hand, from the adaptation law given in (9) the estimate value $\hat{\psi}(t)$ of the uncertain parameter $\psi^{*}$ is also uniformly bounded since the error $\|e(t)\|$ is bounded. Thus, we can complete the proof of this theorem. $\nabla \nabla$

Remark 3.1. In the proof of Theorem 3.1, it is assumed that the positive constant $\varepsilon$ should satisfy some conditions. However, the adaptive robust state observers proposed in (8) with (9) is completely independent of such a constant. Thus, it is not necessary for the system designer to know or choose this constant. In fact, the adaptive robust state observers proposed in (8) with (9) can adjust automatically to counter the destabilizing effects of the uncertainties, delayed state perturbations, and external disturbances.

Remark 3.2. In the light of the definition of the positive constant $\bar{\varepsilon}$, it can be observed that by decreasing sufficiently the value of $\sigma$ which is an adjustable parameter of the adaptation law, one can obtain the radius $\delta$ of the ball as small as desired. Thus, the system designer can tune the size of the residual set by adjusting properly the 
parameter $\sigma$ which is introduced in the adaptation law described by (9).

\section{Illustrative Example}

To illustrate the utilization of our approach, in this section, we consider the following numerical example.

$$
\begin{aligned}
\frac{d x(t)}{d t} & =\left[\begin{array}{rr}
-1 & 2 \\
0 & -3
\end{array}\right] x(t)+\left[\begin{array}{l}
1 \\
1
\end{array}\right] u(t)+\Delta f(x(t-h(t))) \\
y(t) & =\left[\begin{array}{ll}
1 & 1
\end{array}\right] x(t)
\end{aligned}
$$

where

$$
\Delta f(x(t-h(t)))=\left[\begin{array}{c}
0 \\
v \sin \left(x_{1}(t-h(t))\right)
\end{array}\right]
$$

and where $v$ is an uncertain parameter.

It is obvious that the pair $(A, C)$ of the systems described by (15) is completely observable. Thus, we can arbitrarily assign the eigenvalues of the matrix $A_{0}=A-K C$. Here, we will select the eigenvalues of the matrix $A_{0}$ as $\left[\begin{array}{ll}-2 & -3\end{array}\right]$. Then, the corresponding gain $K$ is given by $K=\left[\begin{array}{ll}1 & 0\end{array}\right]^{T}$, and the matrix $A_{0}$ is given by

$$
A_{0}=\left[\begin{array}{rr}
-2 & 1 \\
0 & -3
\end{array}\right]
$$

Now, we have to find a matrix $Q$ such that Assumption 2.2 is satisfied. For this, from (5) we can have that

$$
Q=\left[\begin{array}{ll}
8 & 3 \\
3 & 4
\end{array}\right]
$$

which results in

$$
P=\left[\begin{array}{ll}
2 & 1 \\
1 & 1
\end{array}\right]
$$

Therefore, from (15) to (18) it can easily be verified that Assumption 2.2 is satisfied, i.e.

$$
\Delta f(x(t-h(t)))=P^{-1} C^{T} \xi(x(t-h(t)))
$$

where

$$
\|\xi(x(t-h(t)))\| \leq \theta^{*}\|x(t-h(t))\|
$$

For (8) and (9), we select the following parameters:

$$
\eta=8.0, \quad \gamma=2.0, \quad \sigma=0.2
$$

Thus, from (8) and (9), we can obtain an adaptive robust state observer with a rather simple structure for this numerical example. It is obvious from Theorem 3.1 that the state estimate $\hat{x}(t)$ of the proposed adaptive robust state observer can converge uniformly ultimately to the original state $x(t)$ of the uncertain time-delay system.

For simulation, we give the uncertain parameter $v=0.5$, and initial conditions as follows.

$$
\begin{aligned}
& x(t)=\left[\begin{array}{ll}
8.0 \cos (t) & 8.0 \cos (t)
\end{array}\right]^{T}, \quad t \in[-\bar{h}, 0] \\
& \hat{x}(0)=\left[\begin{array}{ll}
2.0 & 3.0
\end{array}\right]^{T} \\
& \hat{\psi}(0)=12.0
\end{aligned}
$$

In addition, for simplicity, the control input will be set to be zero, i.e. $u(t) \equiv 0$.

With the chosen parameter settings above, the results of simulation are shown in Fig. 1 and Fig. 2 for this numerical example.

It can be observed from Fig. 1 that the state estimate $\hat{x}(t)$ of the proposed adaptive robust state observer indeed converges uniformly ultimately to the original state $x(t)$ of the time-delay system described by (15) and (16). On the other hand, it can be known from Fig. 2 that similar to the conventional adaptation law with sigma-modification, the improved one makes indeed the estimate values of the unknown parameters decreasing. (The figures of the simulation will be displayed in the presentation.)

\section{Concluding Remarks}

The problem of adaptive robust state observers has been considered for a class of uncertain systems with timevarying delays. A new method has been presented whereby a class of continuous memoryless adaptive robust state observers with a rather simpler structure is constructed. Since our adaptive state observers do not involve the upper bound of uncertainties, such upper bound is not required to be known for the system designer. It has been also shown that the proposed adaptive robust state observers can guarantee that the observation error between the observer state estimate and the true state converges uniformly exponentially towards a ball which can be as small as desired.

In addition, because the adaptive robust state observers proposed in the paper are completely independent of time delays, the results obtained here may be applicable to a class of dynamical systems with uncertain time delays. 


\section{Acknowledgment}

This work was supported in part by the Japan Society for the Promotion of Science (JSPS) under Grant (C)22560405.

\section{REFERENCES}

[1] B.L. Walcott and S.H. Zak, "State observation of nonlinear uncertain dynamical systems," IEEE Trans. Automat. Contr., vol.32, 166-170, 1987.

[2] D.M. Dawson, Z. Qu, and J.C. Carroll, "On the state observation and output feedback problems for nonlinear uncertain dynamic systems," Syst. Contr. Let., vol.18, 217-222, 1992.

[3] D.W. Gu and F.W. Poon:, "A robust state observer scheme, " IEEE Trans. Automat. Contr., vol.46, 19581963, 2001.

[4] J. Leyva--Ramos and A.E. Pearson, "An asymptotic modal observer for linear autonomous time lag systems," IEEE Trans. Automat. Contr., vol.40, 1291$1294,1995$.
[5] H. Trinh and M. Aldeen, "Comments on An asymptotic modal observer for linear autonomous time lag systems," IEEE Trans. Automat. Contr., vol.42, 742745, 1997.

[6] $H_{H} \mathrm{H}$. Choi and M.J. Chung, "Robust observer-based $H_{\infty}$ controller design for linear uncertain time-delay systems, " Automatica, vol.33, 1749--1752, 1997.

[7] M. Darouach, "Reduced-order observers for linear neutral delay systems," IEEE Trans. Automat. Contr., vol.50, 1407--1413, 2005.

[8] Y.H. Chen, "Adaptive robust observers for non-linear uncertain systems," International Journal of Systems Science, vol.21, 803--814, 1990.

[9] H. $\mathrm{Wu}$ and T. Tanaka, "Adaptive robust asymptotic state observers for uncertain nonlinear dynamical systems," IEEJ Trans. Electr. Informat. Syst., vol.127, 726--732, 2007.

[10] H. Wu, "Memoryless Adaptive Robust Asymptotic State Observers for a Class of Nonlinear Time--Delay Systems," IET Control Theory Appl., vol.3, 843--851, 2009. 